\title{
OPERATOR EQUATIONS, SEPARATION OF VARIABLES AND RELATIVISTIC ALTERATIONS
}

\author{
ROBERT A. HERMANN \\ Mathematics Department \\ United States Naval Academy \\ Annapolis MD 21403-5002 USA \\ (Received July 27, 1993)
}

\begin{abstract}
In this paper, a simple and unified method is developed that predicts the relativistic alterations of physical measures when the behavior of a natural system is characterized by means of a specific operator equation. Separation of variables is the simple underlying procedure.
\end{abstract}

KEY WORDS AND PHRASES. Gravitational redshift, transverse Doppler effect, mass alteration, general and special relativity, separation of variables, partial differential equations.

1992 AMS SUBJECT CLASSIFICATION CODES. 83A05, 83C99, 35A08.

\section{INTRODUCTION.}

It is often a difficult problem to find a simple and unified mathematical approach to physical science derivations. The term "derivation" refers to the somewhat informal "proof" method used within the physical sciences that might be formalized when physical axioms are specifically included. Often the relativistic alterations in physical measures are obtained by means of essentially different derivations that are somewhat ad hoc in character and may appear to have no simple underlying approach. With respect to natural system behavior that can be characterized by means of a special operator equation, a unifying approach seems to exist.

In a typical undergraduate differential equations course, the method of separation of variables is introduced in a first attempt to solve the one dimensional heat or wave partial differential equation. This same approach may be the unifying factor that allows one to derive the relativistic alterations for all natural system measures that are modeled by a specific operator equation. Further, from the mathematicians point of view, a derivation in generalized form would be the most appropriate.

First, consider the Schwarzchild metric

$$
d S^{2}=\lambda\left(c d t^{m}\right)^{2}-(1 / \lambda)\left(d R^{m}\right)^{2}-\left(R^{m}\right)^{2}\left(\sin ^{2} \theta^{m}\left(d \phi^{m}\right)^{2}+\left(d \theta^{m}\right)^{2}\right)
$$

where, as usual, $\lambda=\left(1-2 G M /\left(c^{2} R^{m}\right)\right), G$ is the gravitational constant, $M$ the mass of a spherically symmetric homogeneous object, $R^{m}$ the radial distance from the center of the object and the superscript $m$ indicates measurements taken for the behavior of a natural system influenced 


\title{
OPERATOR EQUATIONS, SEPARATION OF VARIABLES AND RELATIVISTIC ALTERATIONS
}

\author{
ROBERT A. HERMANN \\ Mathematics Department \\ United States Naval Academy \\ Annapolis MD 21403-5002 USA \\ (Received July 27, 1993)
}

\begin{abstract}
In this paper, a simple and unified method is developed that predicts the relativistic alterations of physical measures when the behavior of a natural system is characterized by means of a specific operator equation. Separation of variables is the simple underlying procedure.
\end{abstract}

KEY WORDS AND PHRASES. Gravitational redshift, transverse Doppler effect, mass alteration, general and special relativity, separation of variables, partial differential equations.

1992 AMS SUBJECT CLASSIFICATION CODES. 83A05, 83C99, 35A08.

\section{INTRODUCTION.}

It is often a difficult problem to find a simple and unified mathematical approach to physical science derivations. The term "derivation" refers to the somewhat informal "proof" method used within the physical sciences that might be formalized when physical axioms are specifically included. Often the relativistic alterations in physical measures are obtained by means of essentially different derivations that are somewhat ad hoc in character and may appear to have no simple underlying approach. With respect to natural system behavior that can be characterized by means of a special operator equation, a unifying approach seems to exist.

In a typical undergraduate differential equations course, the method of separation of variables is introduced in a first attempt to solve the one dimensional heat or wave partial differential equation. This same approach may be the unifying factor that allows one to derive the relativistic alterations for all natural system measures that are modeled by a specific operator equation. Further, from the mathematicians point of view, a derivation in generalized form would be the most appropriate.

First, consider the Schwarzchild metric

$$
d S^{2}=\lambda\left(c d t^{m}\right)^{2}-(1 / \lambda)\left(d R^{m}\right)^{2}-\left(R^{m}\right)^{2}\left(\sin ^{2} \theta^{m}\left(d \phi^{m}\right)^{2}+\left(d \theta^{m}\right)^{2}\right)
$$

where, as usual, $\lambda=\left(1-2 G M /\left(c^{2} R^{m}\right)\right), G$ is the gravitational constant, $M$ the mass of a spherically symmetric homogeneous object, $R^{m}$ the radial distance from the center of the object and the superscript $m$ indicates measurements taken for the behavior of a natural system influenced 
Consequently, $\gamma \lambda^{s}=\lambda^{m}$

Suppose that $T=\Psi$ is the total wave function, $D$ is the operator $\nabla^{2}-p$, where $n=3$, and the constants $k, p$ are those associated with the classical time-dependent Schrödinger equation for an atomic system as it appears in Evans [2, p. 56]. It is not assumed that such a Schrödinger type equation predicts any other behavior except that it reasonably approximates the discrete energy levels associated with atomic system radiation and that the frequency of such radiation may be obtained, at least approximately, from the predicted energy variations. The eigenvalues for this separable solution are corresponded to energy levels $E^{s}$ and $E^{m}$ for such a radiating atomic system. Thus $\gamma E^{s}=E^{m}$. Radiation occurs when there is a discrete change in the energy levels. This yields

$$
\gamma \Delta E^{s}=\Delta E^{m} .
$$

Now simply divide (2.3) by the Planck constant and obtain the basic gravitational frequency redshift expression $\gamma \nu^{s}=\nu^{m}$ as stated in Bergmann [1, p. 222].

Since this actual derivation is slightly generalized, other operator expressions can be substituted for $D$. For another prediction, consider substituting the Laplacian $\nabla^{2}$ for $D$. This would yield for an appropriate object an alteration due to the gravitational field of the usual temperature function obtained when the PDE for internal heat transfer is solved.

\section{ADDITIONAL APPLICATIONS.}

Consider the special theory linear effect line element

$$
d S^{2}=\lambda\left(c d t^{m}\right)^{2}-(1 / \lambda)\left(d r^{m}\right)^{2},
$$

where $\lambda=\left(1-v^{2} / c^{2}\right)$ and $v$ is a constant relative velocity. Suppose a special theory relativistic effect is considered to take place within an atomic system itself and is assumed to be the same effect whether motion is transverse or receding or approaching the observer, then this is modeled with respect to special theory effects by letting $d r^{m}=d r^{3}=0$ in (1.2) and (3.1). Hence (1.4) holds for this physical scenario. Now the same argument used to obtain the gravitational redshift can be applied in order to obtain the relativistic (i.e. transverse Doppler) redshift prediction $\gamma \nu^{s}=\nu^{m}$. Ives and Stilwell [3] were the first to experimentally verify this prediction.

Finally, consider a freely moving particle of mass $M$ moving in a "straight" line with constant relative velocity $v_{E}$. For a Hamilton characteristic function $S^{\prime}$, the classical Hamilton-Jacobi equation becomes $\left(\partial S^{\prime} / \partial r\right)^{2}=-2 M\left(\partial S^{\prime} / \partial t\right)\left[4\right.$, p. 451]. Suppose that $S^{\prime}(r, t)=h(r) f(t)$. Again consider line elements (1.2) and (3.1) while letting the universal nature of $S^{\prime}$ and invariance of separation imply that $h\left(r^{s}\right)=H\left(r^{m}\right), f\left(t^{s}\right)=F\left(t^{m}\right)$. The same argument used for the relativistic redshift derivation yields equation (1.4). Let $D=(\partial(\cdot) / \partial r)^{2}$. The same procedure used to obtain (2.1) and (2.2) yields

$$
\begin{gathered}
\left(\frac{\partial h\left(r^{s}, t^{s}\right)}{\partial r^{s}}\right)^{2}\left(\frac{1}{h\left(r^{s}\right)}\right)=-2 \frac{M^{s}}{f^{2}\left(t^{s}\right)} \frac{d f}{d t^{s}}=M^{s} \lambda_{1}^{s}= \\
-2 \frac{M^{s}}{F^{2}\left(t^{m}\right)} \frac{d F}{d t^{m}} \frac{d t^{m}}{d t^{s}}=M^{s} \lambda_{1}^{m} / \gamma .
\end{gathered}
$$

With respect to $m$,

$$
\left(\frac{\partial H\left(r^{m}, t^{m}\right)}{\partial r^{m}}\right)^{2}\left(\frac{1}{H\left(r^{m}\right)}\right)=-2 \frac{M^{m}}{F^{2}\left(t^{m}\right)} \frac{d F}{d t^{m}}=M^{m} \lambda_{1}^{m} .
$$


In (3.2) and (3.3), the quantities $M^{s}$ and $M^{m}$ are obtained by means of an identical mode of measurement that characterizes "mass." Assuming that the two separated forms in (3.2) and (3.3) are invariant, leads to the special theory mass expression $M^{m}=(1 / \gamma) M^{s}$. These examples amply demonstrate the utility of the separation of variables approach in obtaining various relativistic alterations in measured physical quantities.

ACKNOWLEDGEMENT This work was partially funded by a grant from the United States Naval Academy Research Council.

\section{REFERENCES}

1. BERGMANN, G. Introduction to the Theory of Relativity, Dover, New York, 1976.

2. EVANS, R. D. The Atomic Nucleus, McGraw-Hill, New York, 1955.

3. IVES, H. and G. STILWELL. Experimental study of the rate of a moving atomic clock, $\underline{\mathrm{J}}$. Opt. Soc. Am., 28 (1939), 215-301.

4. SYNGE, J. L. and B.A. GRIFFITH, Principles of Mechanics, McGraw-Hill, New York, 1959. 


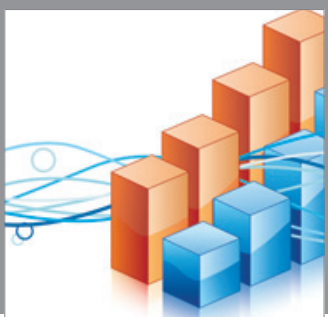

Advances in

Operations Research

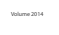

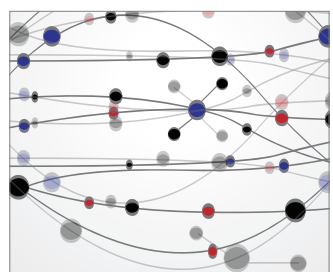

\section{The Scientific} World Journal
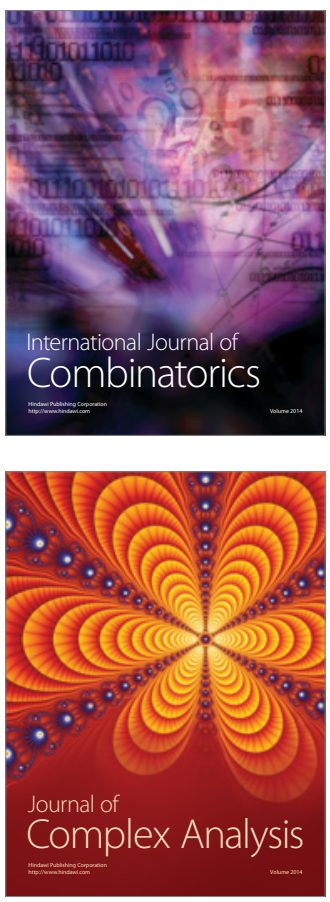

International Journal of

Mathematics and

Mathematical

Sciences
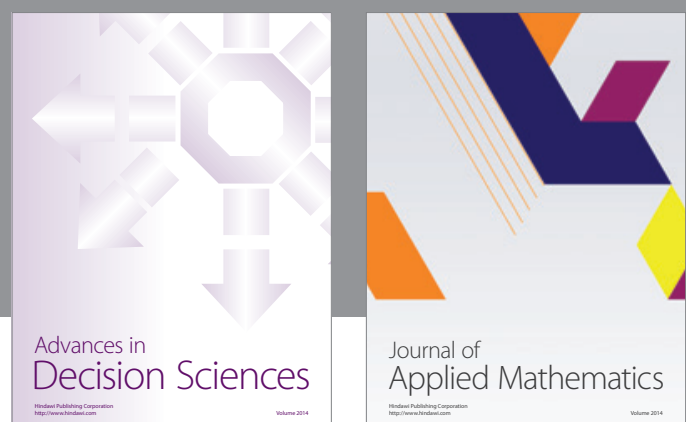

Journal of

Applied Mathematics
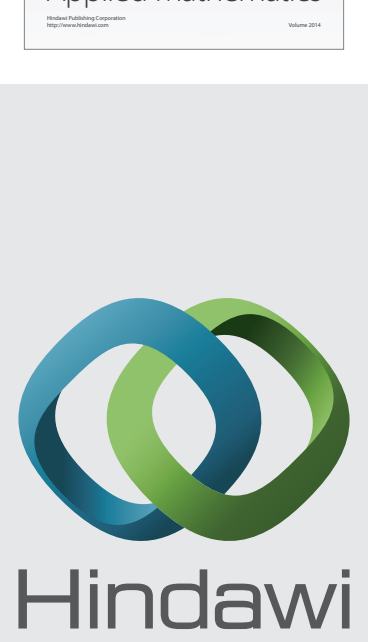

Submit your manuscripts at http://www.hindawi.com
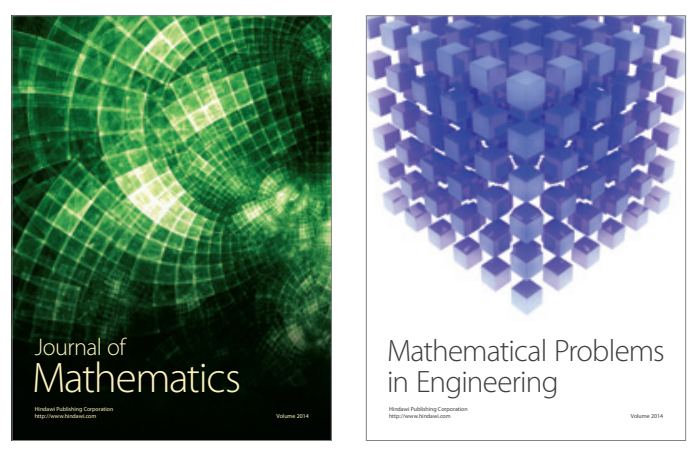

Mathematical Problems in Engineering
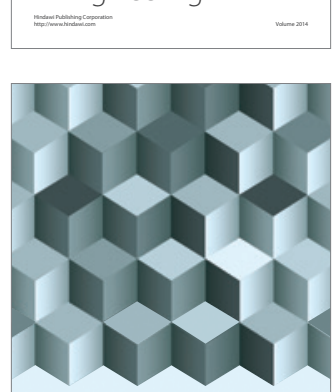

Journal of

Function Spaces
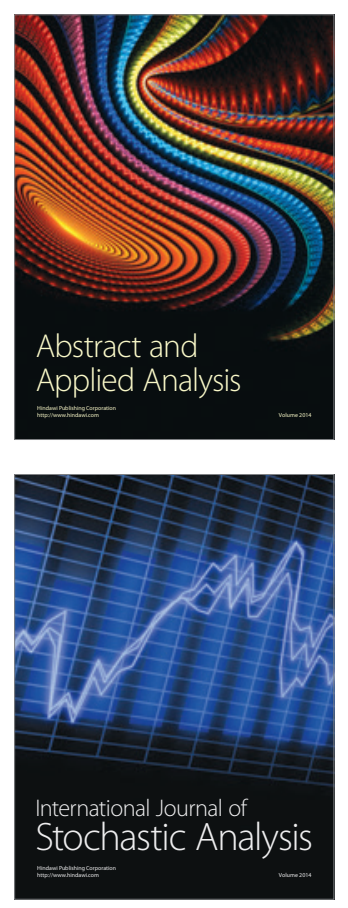

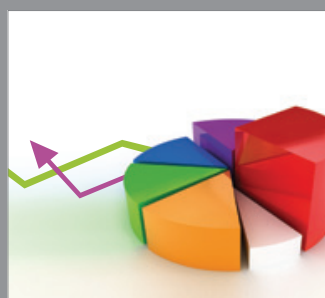

ournal of

Probability and Statistics

Promensencen
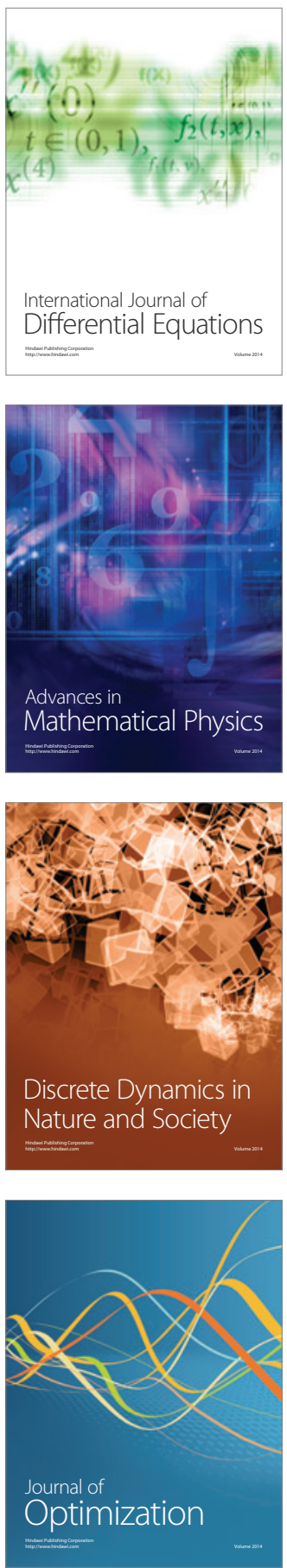